\title{
Antigens containing TAVSPTTLR tandem repeats could be used in assaying antibodies to Classical swine fever virus
}

\author{
Y. QI ${ }^{1,2}$, B.Q. ZHANG ${ }^{1,2}$, Z. SHEN ${ }^{1,2}$, Y.H. CHEN ${ }^{1,2 *}$ \\ ${ }^{1}$ Laboratory of Immunology, Department of Biology, Tsinghua University, Beijing, 100084, P.R. China; ${ }^{2}$ Protein Science Laboratory \\ of MOE, Beijing, 100084, P.R. China
}

Received March 6, 2009; accepted September 9, 2009

\begin{abstract}
Summary. - Classical swine fever virus (CSFV) causes a highly contagious and often fatal viral disease in pigs. The highly conserved epitope TAVSPTTLR on the glycoprotein E2 was shown to be suitable for differentiation of CSFV from other pestiviruses. In this study, we found that swine CSFV antisera contained TAVSPTTLR-related rather than TAVSPTTLR-specific CSFV antibodies. The CSFV antisera reacted only to some extent with a synthetic TAVSPTTLR-containing peptide, but inhibited the binding of TAVSPTTLR-specific antibodies to the viral antigen. Since chimeric antigens containing TAVSPTTLR tetramers or hexamers were recognized by the swine CSFV antisera, such antigens could be potentially applied to the detection of CSFV antibodies. These results might be helpful in designing a TAVSPTTLR epitope-based CSFV vaccine and a corresponding serological test.
\end{abstract}

Keywords: Classical swine fever virus; E2 glycoprotein; TAVSPTTLR epitope; tandem repeats; antibodies

\section{Introduction}

CSFV causes classical swine fever, a highly contagious and often fatal viral disease with devastating impact on the pig industry (Edwards et al., 2000; Moennig, 2000; Stegeman et al., 2000; Paton and Greiser-Wilke, 2003). CSFV is a single-stranded RNA virus belonging to the genus Pestivirus in the family Flaviviridae (Fauquet et al., 2005). The viral genome contains ORF encoding a 3898 aa polypeptide (Meyers et al., 1989; Moormann et al., 1990) that is processed by viral and cellular proteases to different structural and non-structural viral proteins (Rumenapf et al., 1993). Among these proteins, glycoprotein E2 is an immunogenic structural protein inducing neutralizing antibodies and conferring protective immunity (Hulst et al., 1993; Konig et al.,

E-mail: chenyh@mail.tsinghua.edu.cn; fax: +8610-62771613. Abbreviations: $\mathrm{CSFV}=$ Classical swine fever virus; DIVA = differentiating infected from vaccinated individuals; GST $=$ glutathione S-transferase; HIAS = hyperimmune antisera; $\operatorname{MAb}(\mathrm{s})=$ monoclonal antibody(ies)
1995; Sanchez et al., 2008). Topological analysis of E2 with a panel of 13 monoclonal antibodies (MAbs) identified four antigenic domains (A, B, C, and D) on two structural units in the N-terminal half of E2. Domains B/C and A can elicit neutralizing antibodies and provide protection against CSFV challenge (Wensvoort, 1989; van Rijn et al., 1996). The sequence of aa 829-837 in the A domain - TAVSPTTLR - was identified by the MAb WH303 as a highly conserved epitope within different CSFV strains (Lin et al., 2000). Later, this epitope was demonstrated as a neutralizing epitope not only by additional two MAbs (a18 and 24/10), but also by the recombinant immunogens harboring this epitope (Liu et al., 2006a,b; Zhang et al., 2006). In our previous studies we found that this epitope as a predefined linear neutralizing epitope might need an essential contributions from its flanking sequences to induce functional epitope-specific antibodies (Qi et al., 2008).

We were interested in the problem whether the recombinant immunogens based on this epitope could be further developed as the candidate for DIVA (differentiating infected from vaccinated individuals) vaccines or marker vaccines. Apparently, the appropriate vaccine should induce a characteristic antibody pattern that would be completely 
different from that induced by the viral infection (van Oirschot, 1999).

To analyze the antibody response of swine CSFV antisera to the TAVSPTTLR sequence and to estimate the feasibility of the antigens containing TAVSPTTLR tandem repeats as diagnostic reagents, we evaluated these antigens in the indirect ELISA and competitive ELISA.

\section{Materials and Methods}

Peptide, recombinant antigens, and sera. The pepE, CTAVSPTTLRGSTAVSPTTLR, was commercially synthesized (SBS Genetech). The construction, expression, and purification of chimeric GST proteins containing TAVSPTTLR tandem repeats were previously described (Qi et al., 2008). Briefly, the DNA sequence coding for TAVSPTTLR was linked one another with the isocaudarner pair of $B a m \mathrm{HI}$ and $B g l \mathrm{II}$. These DNA sequences were then inserted into pGEX-6p-1 vector (GE Healthcare) and resulting plasmids expressed chimeric GST proteins containing TAVSPTTLR tandem repeats signed GST-e, GST-2e, GST-4e, and GST-6e, respectively (Fig. 2a). The plasmids were transformed into E. coli strain Rosetta. The proteins were induced at $16^{\circ} \mathrm{C}$ by 0.16 $\mathrm{mmol} / \mathrm{l} \mathrm{IPTG}$ for $7 \mathrm{hrs}$ and purified by a standard procedure with glutathione Sepharose 4B column (GE Healthcare). GST protein coded by pGEX-6p-1 was also expressed and purified.

The swine CSFV hyperimmune antisera (HIAS) were kindly provided by Prof. Zai-shi Wang from the China Institute of Veterinary Drug Control. The HIAS were prepared from pigs, which received first inoculation with the CSFV vaccine strain $C$, and then they were challenged with the virulent CSFV strain Shimen. The field sera were randomly collected at a pig farm in Hubei Province. These field sera were designated as Hubei-A, Hubei-B, Hubei-C, Hubei-D, and Hubei-E. The IDEXX CSFV Antibody Test Kit (IDEXX Scandinavia AB) was used to check the presence of the CSFV-specific antibodies in swine sera.

The rabbit CSFV hyperimmune sera (HIAS) were prepared from rabbits inoculated with high-dose of CSFV C strain repetitively and used as a positive control in the competitive ELISA described below (Qi et al., 2008).

Purification of epitope-specific antibodies. The pepE was coupled to NHS-activated Sepharose 4 Fast Flow beads (GE Healthcare) according to the manufacturer's instructions. Using this affinity chromatography, the TAVPSTTLR-specific antibodies in the rabbit HIAS could be purified by elution with the low-pH buffer $(0.1 \mathrm{~mol} / \mathrm{l}$ glycine, $\mathrm{pH} 2.5)$. The purified antibodies were extensively dialyzed against PBS.

Indirect ELISA and TAVSPTTLR-specific antibody competitive ELISA. The indirect ELISA and the TAVSPTTLR-specific antibody competitive ELISA were performed as described (Qi et al., 2008). The pepE and the TAVSPTTLR chimeric GST proteins were used in the binding ELISA. The recombinant E2 A/D domain and pepE were used in the competitive ELISA. For the indirect binding ELISA, the plates coated with antigens were incubated with swine CSFV antisera at different dilutions before the HRPconjugated polyclonal rabbit anti-swine immunoglobulins (DAKO) were added. For the competitive ELISA, the plates coated with antigens were incubated with swine CSFV antisera (rabbit serum without CSFV-specific antibodies as a negative control and rabbit HIAS as a positive control) before the HRP-conjugated TAVSPTTLR-specific antibodies were added and allowed to bind. $\mathrm{A}_{490}$ was read by a Bio-Rad Model 680. The inhibition (\%) was calculated according to the following formula. If the inhibition of the serum was higher than $40 \%$, the serum was considered as inhibiting the reaction between the TAVSPTTLR-specific antibodies and the coating antigen.

$$
\text { Inhibition }(\%)=\frac{\mathrm{A}_{490_{\text {neg }}}-\mathrm{A}_{490_{\text {test }}}}{\mathrm{A}_{490 \mathrm{neg}}} \times 100
$$

SDS-PAGE and Western blot analysis. SDS-PAGE (12.5\% gel) of the chimeric proteins (GST, GST-e, GST-2e, GST-4e and GST-6e) was performed and the proteins were blotted to the nitrocellulose membrane. The rabbit TAVSPTTLR-specific antibodies $(0.07 \mu \mathrm{g} / \mathrm{ml})$ and the swine HIAS $(1: 100)$ were used as the primary antibodies. The HRP-conjugated polyclonal goat anti-rabbit immunoglobulins and the HPR-conjugated polyclonal rabbit anti-swine immunoglobulins (DAKO) were used as secondary antibodies.

\section{Results}

The swine CSFV antisera inhibited TAVSPTTLR-specific antibodies binding to the recombinant E2 A/D domain

The swine sera were first tested by the IDEXX CSFV Antibody Test kit. Besides the hyperimmune sera (HIAS), the field sera Hubei-A and Hubei-E were also scored as CSFV-antibody positive. The field sera Hubei-B, Hubei$\mathrm{C}$, and Hubei-D were CSFV-antibody negative. Then, the HIAS, Hubei-A, and Hubei-E sera were tested in the binding ELISA with the pepE as the coating antigen. The result showed that the swine CSFV antisera did not exhibit any significant binding activity with the pepE even at the dilution 1:20 (data not shown).

Next, the competitive ELISA was used to test the swine CSFV antisera (Fig. 1). Since these antisera did not react with the synthetic TAVSPTTLR peptide, it was not a surprise that they did not inhibit the binding of TAVSPTTLR-specific antibodies to pepE. Unexpectedly, these antisera inhibited the binding of the TAVSPTTLR-specific antibodies to the recombinant E2 A/D domain.

The swine CSFV antisera recognized TAVSPTTLR chimeric GST proteins in a different pattern compared with the TAVSPTTLR-specific antibodies

To further characterize the antibodies in swine CSFV antisera, we designed a few chimeric GST proteins containing TAVSPTTLR tandem repeats and the individual TAVSPTTLR epitope was spaced by a Glycine-Serine adaptor (Fig. 2a). These proteins were successfully produced and tested in SDS-PAGE (Fig. 2b). Western blot analysis was performed 
using the swine CSFV antisera or the TAVSPTTLR-specific antibodies as primary antibodies. The results showed that the TAVSPTTLR-specific antibodies recognized GST-e, GST-2e, GST-4e and GST-6e antigens, but not GST (Fig. 2c). Similar to the TAVSPTTLR-specific antibodies, the swine HIAS recognized GST-4e, GST-6e antigens, but not GST. On the other hand, the HIAS did not react with GST-e and GST-2e showing a different recognition pattern (Fig. 2d). The field sera Hubei-A and Hubei-E showed the similar binding pattern as the HIAS (data not shown).

Two chimeric GST proteins reacted with CSFV antibodies in swine sera.

A series of binding ELISAs were performed with microtiter plates coated with different chimeric antigens. The HIAS and the field sera were used as the primary antibodies at different dilutions (Fig. 3). As a result, the swine CSFV antisera and the negative sera reacted with GST-e and GST-2e in a rather similar pattern (Fig. 3a,b), while they displayed a significantly different pattern, when reacting with GST-4e and GST-6e (Fig. 3c,d).

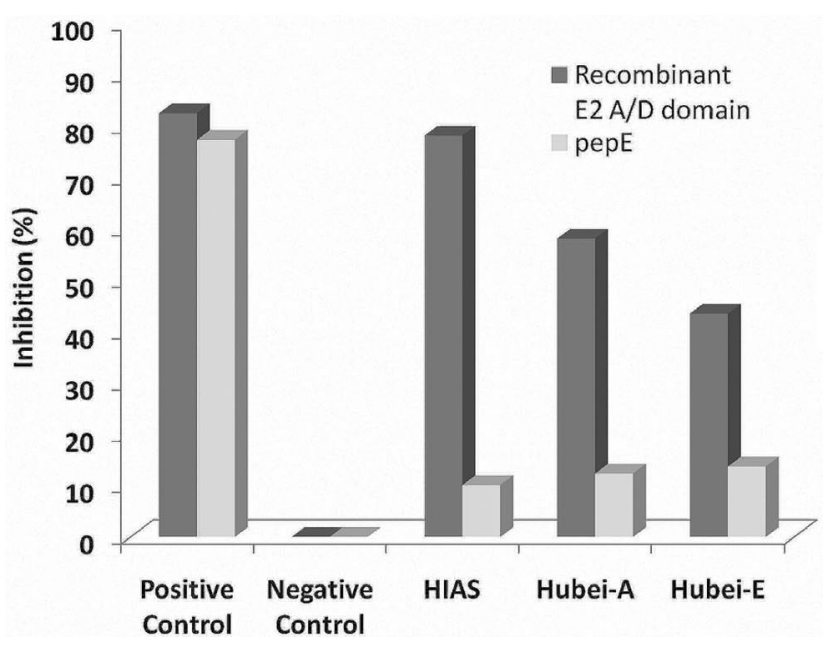

Fig. 1

Inhibition of the binding of TAVSPTTLR-specific antibodies to CSFV antigen by CSFV antisera

HIAS, Hubei-A, and Hubei-E were positive for CSFV-specific antibodies. Positive control $=$ rabbit HIAS; negative control $=$ rabbit serum without CSFV-specific antibodies.

(a)

GST GST-LGSPEFPGRLERPHR

GST-e GST-LGSTAVSPTTLRGS

GST-2e GST-LGSTAVSPTTLRGSTAVSPTTLRGS

GST-4e GST-LGSTAVSPTTLRGSTAVSPTTLRGSTAVSPTTLRGSTAVSPTTLRGS

GST-6e GST-LGSTAVSPTTLRGSTAVSPTTLRGSTAVSPTTLRGSTAVSPTTLRGSTAVSPTILRGSTAVSPTTLRGS

(b)

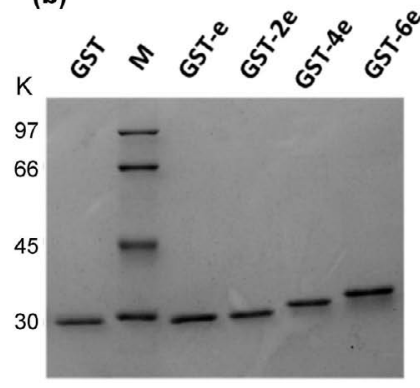

(c)

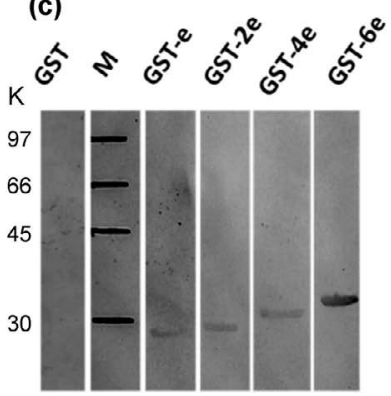

(d)

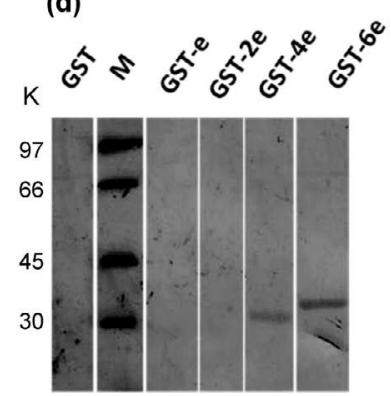

Fig. 2

Chimeric GST proteins containing TAVSPTTLR epitope (a), their SDS-PAGE profiles (b) and Western blot analysis using TAVSPTTLRspecific antibodies (c) and HIAS (d)

$\mathrm{M}=$ protein size marker. 
(a)

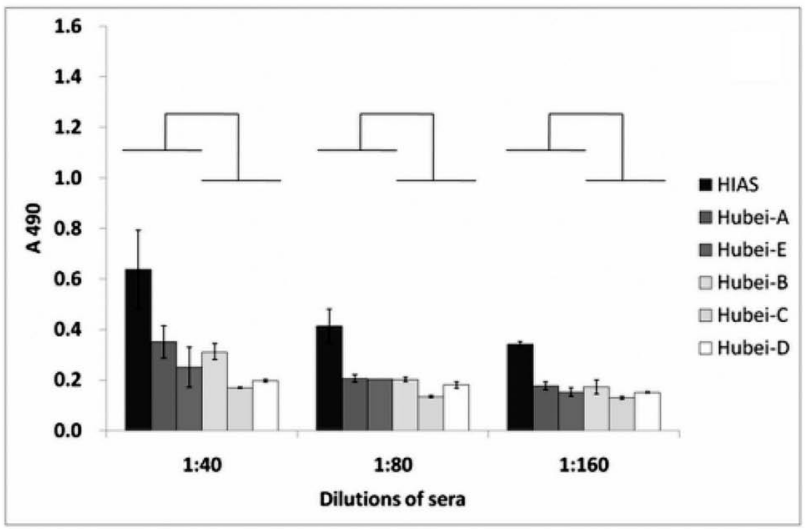

(c)

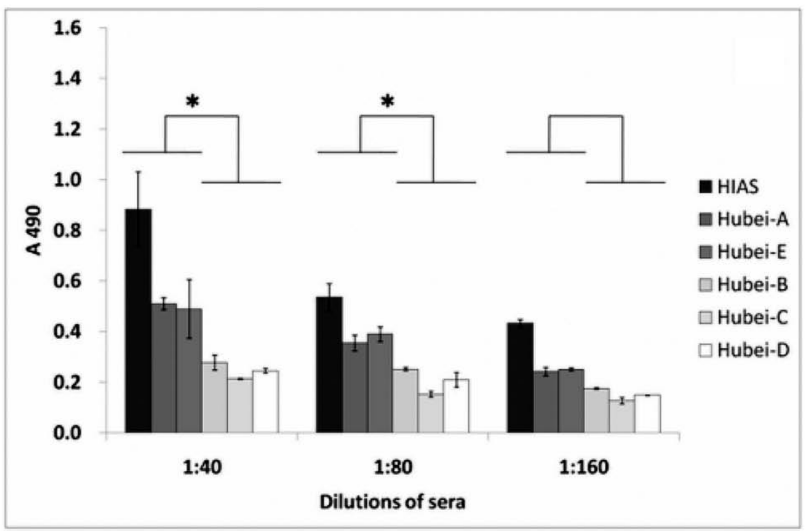

(b)

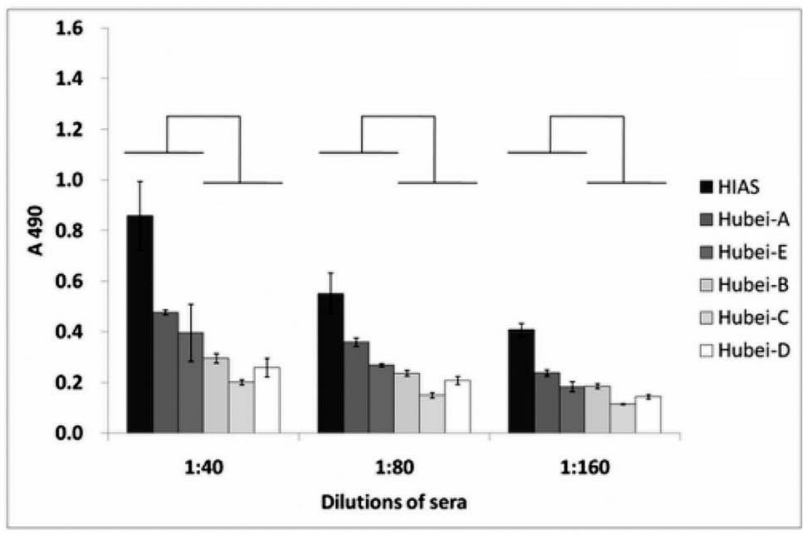

(d)

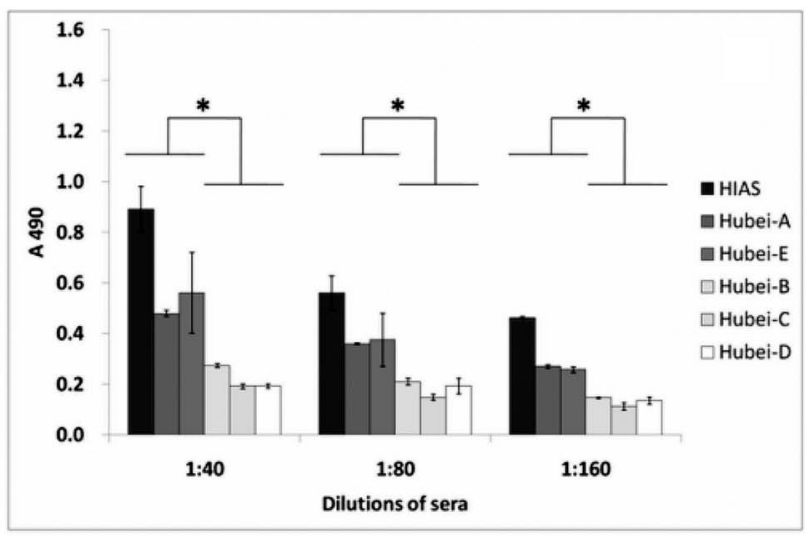

Fig. 3

Binding ELISA of CSFV antisera to chimeric GST proteins containing TAVSPTTLR epitope

Coating antigens: GST-e (a), GST-2e (b), GST-4e (c), and GST-6e (d). HIAS, Hubei-A, and Hubei-E were positive for CSFV antibodies, while Hubei-B, Hubei-C, and Hubei-D were negative. The asterisk (*) indicated a statistically significant difference.

\section{Discussion}

In our previous studies with the synthetic peptide pepE as the coating antigen in ELISA, we found a significant titer of TAVSPTTLR-specific antibodies in the rabbit CSFV antisera (about 1:1280 1:2560). We also purified these epitopespecific antibodies by immunoaffinity chromatography (Qi et al., 2008). In this report, we used the same ELISA to test the swine CSFV antisera including HIAS and field sera. However, the swine CSFV antisera did not show significant binding activities with the pepE. Thus, it was indicated that the epitope TAVSPTTLR was conserved and accessible for the epitope-specific neutralizing antibodies. However, it displayed a different immunogenicity in different animals. CSFV virions induced TAVSPTTLR-specific antibodies in the rabbits, but did not induce such antibodies in the pigs. This finding was in agreement with the fact that polyclo- nal IgG from pig hyperimmune sera did not react with the CSFV peptide aa 804-910, which covered the TAVSPTTLR epitope (Lin et al., 2000). This information might be useful for the epitope-based DIVA vaccine design, since the virions did not induce TAVSPTTLR-specific antibodies while the recombinant immunogen did (Liu et al., 2006a,b).

Interestingly, though the swine CSFV antisera did not inhibit the binding of the TAVSPTTLR-specific antibodies to the pepE, they managed to inhibit the binding of such epitope-specific antibodies to the recombinant A/D domain of the CSFV E2 glycoprotein. Therefore, we hypothesized that there were TAVSPTTLR-related antibodies in the swine CSFV antisera. Although they could compete with the TAVSPTTLR-specific antibodies for the binding site on the CSFV antigens, they were not identical to the TAVSPT TLR-specific antibodies. The different recognition patterns for swine CSFV antisera and TAVSPTTLR-specific antibod- 
ies demonstrated the presence of the TAVSPTTLR-related antibodies in the swine CSFV antisera.

The TAVSPTTLR chimeric GST proteins were candidate for the CSFV marker vaccines. Hence, one of the essential requirements for chimeric antigens is their ability to differentiate between presence or absence of CSFV antibodies in the pig sera. Therefore, we compared the recognition patterns of CSFV-antibody positive or negative sera in reaction with TAVSPTTLR chimeric proteins. The results showed that all TAVSPTTLR chimeric proteins were not suitable for the detection of CSFV antibodies. The antigens GST-4e and GST-6e were adequate for detection of CSFV antibodies, but the antigens GST-e and GST-2e were not.

In previous studies, two types of subunit marker vaccines and their corresponding diagnostic methods were described. The first vaccine was based on the two antigenic units of the E2 protein (van Rijn et al., 1999) and the second one was based on the antibody responses directed to the E2 and $\mathrm{E}^{\text {rns }}$ protein (Moormann et al., 2000; Floegel-Niesmann, 2001). In this study, we found the presence of TAVSPT TLR-related but not TAVSPTTLR-specific antibodies in the swine CSFV antisera. Therefore, the TAVSPTTLR -specific antibodies induced by the TAVSPTTLR-based marker vaccines could be used as a detecting marker. Since GST-4e and GST-6e antigens were suitable for the detection of CSFV antibodies in pig sera, a combination of a TAVSPTTLR-harboring peptides (i.e. pepE, GST-e, or GST-2e) and GST-4e or GST-6e might be applied in the serological test associated with a TAVSPTTLR-based marker vaccine. Therefore, this study might be helpful for the design of a TAVSPTTLR-based vaccine and the corresponding serological test.

Acknowledgements. The authors thank Prof. Zai-Shi Wang and Mr. Lei Wu, Institute of Veterinary Drug Control, P.R. China, for help in the animal experiments and Zhao Chen, Tsinghua University, Beijing, P.R. China, for help in the preparation of this manuscript. This study was supported by the grant 9732007CB914402 from the Ministry of Science and Technology of the People's Republic of China and by the funds from the Beijing Feikai Biotech Ltd.

\section{References}

Edwards S, Fukusho A, Lefevre PC, Lipowski A, Pejsak Z, Roehe P, Westergaard J (2000): Classical swine fever: the global situation. Vet. Microbiol. 73, 103-119. doi:10.1016/ $\underline{\text { S0378-1135(00)00138-3 }}$

Fauquet CM, Mayo MA, Maniloff J, Desselberger U, Ball LA (2005): Virus Taxonomy. Eighth Report of the International Committee on Taxonomy of Virus. Elsevier-Academic Press, Ameserdam, pp. 981-998.

Floegel-Niesmann G (2001): Classical swine fever (CSF) marker vaccine. Trial III. Evaluation of discriminatory ELISAs.
Vet. Microbiol. 83, 121-136. doi:10.1016/S03781135(01)00411-4

Hulst MM, Westra DF, Wensvoort G, Moormann RJ (1993): Glycoprotein E1 of hog cholera virus expressed in insect cells protects swine from hog cholera. J. Virol. 67, 5435-5442.

Konig M, Lengsfeld T, Pauly T, Stark R, Thiel HJ (1995): Classical swine fever virus: independent induction of protective immunity by two structural glycoproteins. J. Virol. 69, 6479-6486.

Lin M, Lin F, Mallory M, Clavijo A (2000): Deletions of structural glycoprotein E2 of classical swine fever virus strain alfort/187 resolve a linear epitope of monoclonal antibody WH303 and the minimal N-terminal domain essential for binding immunoglobulin $\mathrm{G}$ antibodies of a pig hyperimmune serum. J. Virol. 74, 11619-11625. doi:10.1128/JVI.74.24.11619-11625.2000

Liu S, Tu C, Wang C, Yu X, Wu J, Guo S, Shao M, Gong Q, Zhu Q, Kong X (2006a): The protective immune response induced by $\mathrm{B}$ cell epitope of classical swine fever virus glycoprotein E2. J. Virol. Methods 134, 125-129. doi:10.1016/j.jviromet.2005.12.008

Liu S, Yu X, Wang C, Wu J, Kong X, and Tu C (2006b): Quadruple antigenic epitope peptide producing immune protection against classical swine fever virus. Vaccine 24 , 7175-7180. doi:10.1016/j.vaccine.2006.06.042

Meyers G, Rumenapf T, Thiel HJ (1989): Molecular cloning and nucleotide sequence of the genome of hog cholera virus. Virology 171, 555-567. doi:10.1016/00426822(89)90625-9

Moennig V (2000): Introduction to classical swine fever: virus, disease and control policy. Vet. Microbiol. 73, 93-102. doi:10.1016/S0378-1135(00)00137-1

Moormann RJ, Bouma A, Kramps JA, Terpstra C, De Smit HJ (2000): Development of a classical swine fever subunit marker vaccine and companion diagnostic test. Vet. Microbiol. 73, 209-219. doi:10.1016/S0378-1135(00)00146-2

Moormann RJ, Warmerdam PA, van der Meer B, Schaaper WM, Wensvoort G, Hulst MM (1990): Molecular cloning and nucleotide sequence of hog cholera virus strain Brescia and mapping of the genomic region encoding envelope protein E1. Virology 177, 184-198. doi:10.1016/00426822(90)90472-4

Paton DJ, Greiser-Wilke I (2003): Classical swine fever--an update. Res. Vet. Sci. 75, 169-178. doi:10.1016/S00345288(03)00076-6

Qi Y, Liu LC, Zhang BQ, Shen Z, Wang J, Chen YH (2008): Characterization of antibody responses against a neutralizing epitope on the glycoprotein E2 of classical swine fever virus. Arch. Virol. 153, 1593-1598. doi:10.1007/s00705$\underline{008-0147-1}$

Rumenapf T, Unger G, Strauss JH, Thiel HJ (1993): Processing of the envelope glycoproteins of pestiviruses. J. Virol. 67, 3288-3294.

Sanchez O, Barrera M, Rodriguez MP, Frias MT, Figueroa NE, Naranjo P, Montesino R, Farnos O, Castell S, Venereo A, Ganges L, Borroto C, Toledo JR (2008): Classical swine fever virus E2 glycoprotein antigen produced in 
adenovirally transduced PK-15 cells confers complete protection in pigs upon viral challenge. Vaccine 26, 988-997. doi:10.1016/j.vaccine.2007.11.014

Stegeman A, Elbers A, de Smit H, Moser H, Smak J, Pluimers F (2000): The 1997-1998 epidemic of classical swine fever in the Netherlands. Vet. Microbiol. 73, 183-196. doi:10.1016/S0378-1135(00)00144-9

van Oirschot JT (1999): Diva vaccines that reduce virus transmission. J. Biotech. 73, 195-205. doi:10.1016/S0168$\underline{1656(99) 00121-2}$

van Rijn PA, Bossers A, Wensvoort G, Moormann, RJ (1996): Classical swine fever virus (CSFV) envelope glycoprotein E2 containing one structural antigenic unit protects pigs from lethal CSFV challenge. J. Gen. Virol. 77, 2737-2745. doi:10.1099/0022-1317-77-11-2737

van Rijn PA, van Gennip HG, de Meijer EJ, Moormann RJ (1993): Epitope mapping of envelope glycoprotein E1 of hog cholera virus strain Brescia. J. Gen. Virol. 74, 2053-2060. doi:10.1099/0022-1317-74-10-2053

van Rijn PA, van Gennip HG, Moormann RJ (1999): An experimental marker vaccine and accompanying serological diagnostic test both based on envelope glycoprotein E2 of classical swine fever virus (CSFV). Vaccine 17, 433-440. doi:10.1016/S0264-410X(98)00215-1

Wensvoort G (1989): Topographical and functional mapping of epitopes on hog cholera virus with monoclonal antibodies. J. Gen. Virol. 70, 2865-2876. doi:10.1099/0022$\underline{1317-70-11-2865}$

Zhang F, Yu M, Weiland E, Morrissy C, Zhang N, Westbury H, Wang LF (2006): Characterization of epitopes for neutralizing monoclonal antibodies to classical swine fever virus E2 and Erns using phage-displayed random peptide library. Arch. Virol. 151, 37-54. doi:10.1007/s00705$\underline{005-0623-9}$ 